\title{
REZENSIONEN
}

\section{Das Untersuchungsausschussgesetz des Bundes: praxisnah kommentiert}

Waldhoff, Christian und Klaus Ferdinand Gärditz: Gesetz zur Regelung des Rechts der Untersuchungsausschüsse des Deutschen Bundestages: PUAG, Kommentar, Verlag C. H. Beck, München 2015, 491 Seiten, $€$ 99,-.

Untersuchungsausschüsse sind ein bewährtes Instrument parlamentarischer Kontrolle. In ihren Möglichkeiten, Beweise zu erheben, ähneln sie einem Gericht. Ein parlamentarischer Untersuchungsausschuss ist jedoch ein politisches Gremium, ein Mittel des politischen Kampfes. Er bewegt sich - wie Bundestagspräsident Norbert Lammert in seinem Vorwort zu dem hier besprochenen Kommentar hervorhebt - zwischen Wahrheitssuche und politischem Tribunal.

Umso wichtiger ist eine zweckmäßige, rechtlich und politisch tragfähige Ausgestaltung des Untersuchungsausschussrechts. Artikel 44 GG gibt dazu zwar mit dem Hinweis auf die Vorschriften des Strafprozesses Anhaltspunkte; aber die Rechte der Minderheit - die das Kontrollinstrument Untersuchungsausschuss in erster Linie, aber keineswegs ausschließlich nutzt - und der Mehrheit müssen auch in den Einzelheiten so austariert werden, dass eine Untersuchung ohne Missbrauch und ohne Blockade durch eine der beiden Seiten stattfinden kann. Zugleich muss das Beweiserhebungsrecht unter Wahrung der Grundrechte von Zeugen und unter Berücksichtigung der Notwendigkeit des Geheimschutzes in sensiblen Bereichen geregelt werden.

Das Untersuchungsausschussgesetz des Bundes (PUAG) trat am 26. Juni 2001 in Kraft, also vor knapp eineinhalb Jahrzehnten. Es wurde seitdem, mit Ausnahme einer redaktionellen Anpassung, nicht geändert. Anwendung fand es seitdem auf das Verfahren von mittlerweile elf Untersuchungsausschüssen. Es hat sich - trotz immer wieder geäußerter Kritik an Einzelpunkten - im Großen und Ganzen bewährt, und auch das Bundesverfassungsgericht hat sich auf Regelungen des PUAG bezogen und sie vor dem Hintergrund des Grundgesetzes als angemessen beurteilt. ${ }^{1}$

Nachdem bereits Monographien und ein Kommentar zum PUAG erschienen sind ${ }^{2}$, legen Waldhoff und Gärditz nunmehr einen Kommentar vor, der Recht und politische Praxis aufs engste miteinander verzahnt. Das zeigt sich zum einen darin, dass sie namhafte Vertreter sowohl aus der Rechtswissenschaft als auch aus der parlamentarischen Praxis als Autoren gewinnen konnten, darunter Abgeordnete und Mitarbeiter aller Fraktionen sowie aus der Verwaltung des Bundestags. Zum anderen zeigt es sich am Inhalt: Der rechtswissenschaftli-

1 Siehe BVerfGE 105, 197, 226, 234 zu $\$ 17$ Abs. 3 PUAG (Reihenfolge der Vernehmung von Zeugen und Sachverständigen).

2 Vgl. nur Butz Peters, Untersuchungsausschussrecht. Länder und Bund, München 2012; Paul Glauben / Lars Brocker, Das Recht der parlamentarischen Untersuchungsausschüsse in Bund und Ländern. Ein Handbuch, Köln 2011; dies., PUAG. Kommentar zum Gesetz zur Regelung des Rechts der Untersuchungsausschüsse des Deutschen Bundestages, Köln 2011; Dieter Wiefelspütz, Das Untersuchungsausschussgesetz, Baden-Baden 2003. 
che Teil des Kommentars wird ergänzt durch einen rechtspolitischen Teil, in dem zu Bewährung und Reformbedarf des PUAG aus Sicht der Bundestagsfraktionen Stellung genommen wird.

Der Kommentar beginnt zunächst mit einer thematisch in fünf Teile gegliederten Vorbemerkung. Knapp und instruktiv schildert Hermann Butzer die Geschichte der parlamentarischen Untersuchung und Matthias Roßbach die des Untersuchungsausschussgesetzes. Christian Waldhoff skizziert die Rechtsquellen des parlamentarischen Untersuchungsrechts, Jelena von Achenbach geht ein auf die Abgrenzung der Untersuchungsrechte im europäischen und deutschen Mehrebenensystem (EU, Bund, Länder, Kommunen) - ein Aspekt, dessen Relevanz gerade bei parallelen Untersuchungen auf Bundes- und Landesebene deutlich wird. Das Verhältnis des Untersuchungsausschussgesetzes zur Strafprozessordnung beleuchtet Klaus Ferdinand Gärditz. Tobias Linke gibt einen Überblick über das Untersuchungsausschussrecht in den Ländern, das sich in manchen Einzelheiten teilweise von dem des Bundes deutlich unterscheidet, etwa indem manche Länder Zeugen, gegen die sich die Untersuchung richtet, einen besonderen Betroffenenstatus einräumen.

Die sich anschließende Kommentierung der einzelnen Paragrafen des PUAG ist aktuell und instruktiv. Die Praxis erhält Antworten auf alle relevanten Fragen rund um das Untersuchungsverfahren. Rechtsprechung und Literatur sind umfassend ausgewertet. Wo es an einer gefestigten Judikatur fehlt, geben die Autoren gut begründete Orientierungshilfe. Dazu zwei Beispiele:

Bei der Kommentierung der Vorschriften zum Schutz der Vertraulichkeit (\$\$ 14 bis 16 PUAG) betont Ute Sacksofsky die hohe Bedeutung der Öffentlichkeit im parlamentarischen Verfahren. Zu Recht kritisiert sie die gelegentliche Neigung der Bundesregierung, Unterlagen vorschnell als „VS-Vertraulich“ zu kennzeichnen und so der öffentlichen Erörterung zu entziehen. In der Vergangenheit führte das zu grotesken Ergebnissen, wenn die gleichen Inhalte in parallelen Ausschüssen auf Landesebene offen behandelt wurden. ${ }^{3}$ Vor diesem Hintergrund überzeugt Sacksofskys Auslegung des $\$ 15$ Abs. 1 PUAG, der so zu verstehen sei, dass der Untersuchungsausschuss Geheimhaltung anordnen müsse, aber auch nur dann anordnen dürfe, wenn kollidierende Verfassungsrechtsgüter es erfordern. Ermessen habe der Untersuchungsausschuss dabei nicht.

Bei der Vernehmung von Amtsträgern ( $\$ 23$ PUAG) ist insbesondere die Beurteilung von Beschäftigten von staatlichen oder staatlich beherrschten Organisationen in privater Rechtsform umstritten. Claudia von Cossel will sie - unter Berufung auf entsprechende strafprozessuale Literatur zu $\$ 54$ StPO - grundsätzlich nicht zu den Amtsträgern zählen, mit der Folge, dass sie keiner Aussagegenehmigung bedürfen. Das erscheint konsequent, da sich der Staat, wenn er sich privater Rechtsformen bedient, insoweit auch freiwillig des Einflusses begibt, den er durch die Entscheidung über eine Aussagegenehmigung im Rahmen des $\$ 23$ in Verbindung mit $\$ 18$ Abs. 1 bis 3 PUAG ausüben könnte, und zwar völlig unabhängig von der Frage, inwieweit privatrechtliche Organisationen des Staates der parlamentarischen Kontrolle unterworfen sind.

Genug der Beispiele für eine rundum gelungene Kommentierung. Abgerundet wird das Werk durch den bemerkenswerten rechtspolitischen Ausblick auf das Untersuchungsausschussgesetz durch Abgeordnete aller in der 17. Wahlperiode vertretenen Bundestagsfrak-

3 Siehe Bericht des Untersuchungsausschusses „DDR-Vermögen“, BT-Drs. 13/10900, S. 393 f. 
tionen. Die ernsthafte Prüfung durch diejenigen, deren Rüstzeug das PUAG in der Praxis bildet, ergibt einige bedenkenswerte Anregungen für Änderungen, etwa im Hinblick auf größere Öffentlichkeit und weniger Geheimhaltung (Max Stadler) oder auf eine Verteilung der Zeiten für die Befragung von Zeugen, die den Interessen der kleinen Oppositionsfraktionen besser gerecht wird (Hans-Christian Ströbele, Jens Lehmann / Petra Pau). Im Übrigen hat sich das Gesetz auch nach Ansicht der Fraktionen offensichtlich weitgehend bewährt.

Den Herausgebern und Autoren ist mit diesem Kommentar ein außerordentlicher Wurf gelungen. Als einziger Kritikpunkt bleibt das Schriftbild, das so klein ist, dass es die Lesefreude ein wenig trübt. Ungeachtet dessen wird der Waldhoff/ Gärditz der Parlamentspraxis in Bund und Ländern ein unentbehrlicher Leitfaden sein. Er bereichert zugleich die wissenschaftliche und die rechtspolitische Diskussion um die Weiterentwicklung des Untersuchungsausschussrechts.

Florian Edinger

\section{Der Verteidigungsausschuss als Untersuchungsausschuss: parlamentsrechtlich und verfassungssystematisch kompetent untersucht}

Hilgers, Hans Anton: Der Verteidigungsausschuss als Untersuchungsausschuss gemäß Art. 45 a Abs. 2 des Grundgesetzes (Schriftenreihe: Brandenburgische Studien zum Öffentlichen Recht), Verlag Dr. Kovac, Hamburg 2015, 409 Seiten, € 129,80.

Angesichts der aktuellen Konjunktur von Untersuchungsbedürfnissen im Deutschen Bundestag erscheint die Studie von Hilgers zum richtigen Zeitpunkt. Das gilt insbesondere für die Abgrenzung der Zuständigkeiten zwischen einem Verteidigungsausschuss, der sich selbst als Untersuchungsausschuss konstituiert, und dem allgemeinen Untersuchungsrecht des Bundestages mittels eines Ausschusses nach Artikel 44 GG. Wer „darf“ oder „muss“ bestimmte Kontrollaufgaben unter Einsatz des parlamentarischen Untersuchungsverfahrensrechts wahrnehmen? Hat der „Verteidigungsausschuss als Untersuchungsausschuss“ ein Monopol für Untersuchungen auf dem gesamten Gebiet der Verteidigung, und wenn ja, was fällt in dieses Gebiet: zum Beispiel das Beschaffungswesen? Oder ist dies dem Budgetrecht des Parlaments zuzuordnen, mit der Folge, dass ein Untersuchungsausschuss nach Art. 44 GG einzusetzen wäre? Der politisch interessante Unterschied liegt unter anderem in der Öffentlichkeit der Beweisaufnahme: Für den Ausschuss nach Art. 44 GG ist sie die Regel, für den untersuchenden Verteidigungsausschuss gilt sie weithin als ausgeschlossen (Art. 45a Abs. 3).

Hilgers' Arbeit nimmt in grundlegender Weise ein Thema auf, das in der Literatur zum Untersuchungsrecht des Bundestages nur wenig Aufmerksamkeit gefunden hat. In der Regel eher beiläufig wird die dem Verteidigungsausschuss verfassungsnormativ eingeräumte Untersuchungsbefugnis mitbehandelt, gleichsam als Unterfall oder Nebenkriegsschauplatz des parlamentarischen Enqueterechts aus Art. 44 GG. Das Literaturverzeichnis zur vorliegenden Arbeit, übrigens sehr umfassend und detailliert, zeigt nur ganz wenige spezialisierte Schriften - im Wesentlichen von Elmar Hucko, Hans-Joachim Berg und Tade Matthias 